with platinum as carrier from $2 N$ hydrochloric acid solution with hydrogen sulphide). We consider the appearance of the $16 \mathrm{~min}$. and $72-76 \mathrm{hr}$. activities (presumably identical with Hahn, Meitner and Strassmann's ${ }^{2} 16 \mathrm{~min}$. eka-rhenium and $66 \mathrm{hr}$. eka-iridium) on the glass plate to be conclusive proof that these substances must be due to recoiling fragments of the bursting uranium nucleus, and that they are not 'trans-uranium' elements coming from uranium by a chain of $\beta$-processes.

We have been looking also for possible 'transuranium' elements resulting from the decay of uranium (23 min.), which is certainly a uranium isotope. Some weak activities which have some of the properties of 'trans-uranium' elements have been found. More rigorous tests are in progress, however, to make their identity certain.

The activities which are obtained by recoil on the glass plates when thorium is bombarded have also been studied. As a great number of periods were observed, we have for the present classified only some of them into groups according to their chemical behaviour. We found, for example, bodies of $80 \mathrm{~min}$., $14 \mathrm{hr}$. and 12 days half-lives with the properties of rare earths (lanthanum carrier), $6 \mathrm{hr}$. with those of barium, $70 \mathrm{~min} ., 4 \frac{1}{2}$ days and about $11 \mathrm{hr}$. (which latter appears to grow from the $4 \frac{1}{2}$-day body) with the properties of noble metals. Blank tests showed that thorium $\mathrm{B}$ and thorium $\mathrm{C}$ activities were negligible.

In addition, from samples of specially purified thorium nitrate irradiated in bulk, activities of $2 \frac{1}{2} \mathrm{hr}$. and $22 \mathrm{hr}$. were extracted which had the properties of bromine. Since the $2 \frac{1}{2} \mathrm{hr}$. period has been assigned ${ }^{3}$ to ${ }^{83} \mathrm{Br}$, it is natural to identify this with our $2 \frac{1}{2} \mathrm{hr}$. period. Two weak selenium activities (some hours and some days) were also observed.

These experiments were possible only through the kind collaboration of the High Tension Laboratory staff, who provided the neutron sources (lithium + deuteron). We are also indebted to Dr. R. S. Krishnan for his aid in taking readings with the counter set. E. Bretscher. L. G. Cook.

Cavendish Laboratory,

Cambridge.

March 11 .

' Joliot, F., C.R., 208, 341 (1939).

${ }^{2}$ Hahn, Meitner and Strassmann, Ber., 69, 905 (1936) ; 70, 1374 (1937).

${ }^{3}$ Snell, A. H., Phys. Rev., 52, 1007 (1937).

\section{Liquefaction of Helium}

ON March 7, we succeeded in liquefying helium by using an apparatus based on the Simon expansion principle. We obtained with one expansion, starting from a pressure of $80 \mathrm{~atm}$. and a temperature of $14 \cdot 6^{\circ} \mathrm{K}$., a quantity of about 65 c.c. of liquid helium. The maximum capacity of our apparatus is about 120 c.c. It was also possible to lower the temperature to $3 \cdot 3^{\circ} \mathrm{K}$., by reducing the pressure above the helium.

We made also one expansion starting from a pressure of $30 \mathrm{~atm}$. and a temperature of $24^{\circ} \mathrm{K}$., and we arrived at a temperature of $11^{\circ} \mathrm{K}$.

It is worth pointing out that the time which is necessary to obtain liquid helium by this method is relatively short. On March 7 we obtained helium in 6 hours. On March 14 we repeated the experiment, and then only 4 hours was required to obtain liquid helium. It will no doubt be possible to reduce this time to only 3 hours. In this time is also reckoned the time which is necessary to liquefy the hydrogen used for this experiment.

During these experiments I was assisted by M. van Dingenen and M. A. Werner.

Physical Laboratory, A. YAN ITTERBEeK. University, Louvain.

March 15.

\section{Effect of Foreign Gases on the Continuous Absorption Spectrum of Bromine}

Although much attention has been devoted to the broadening of spectral lines in the presence of foreign gases, little systematic work has been done on the effect of foreign gases on intensities of absorption spectra. We have therefore examined the effect of the following gases on the continuous absorption of bromine, using an objective method of photographic photometry : hydrogen chloride and bromide, carbon dioxide, oxygen and nitrogen.

With bromine and the foreign gas at partial pressures of $2 \mathrm{~cm}$. and $60 \mathrm{~cm}$. respectively, the effect is to increase the intensity of the main absorption continuum $\left(\lambda_{\max }=4150 \mathrm{~A}\right.$.) without altering the general shape of the absorption curve. The $A$ and $B$ components of this continuum ${ }^{1}$ are affected about equally, and there is practically no shift in the position of the maximum. The table of absorption coefficients at the maximum serves to show the magnitude of the effect.

\begin{tabular}{|c|c|c|c|c|c|c|}
\hline Gas & $\begin{array}{c}\text { Bromine } \\
\text { alone }\end{array}$ & \multicolumn{4}{|c|}{ Bromine plus $60 \mathrm{~cm}$. of } \\
\cline { 3 - 6 } & $\mathrm{HCl}$ & $\mathrm{CO}$ & $\mathrm{HBr}$ & $\mathrm{O}_{2}$ & $\mathrm{~N}_{2}$ \\
\hline$\varepsilon_{\max .}$ & 152 & 191 & 184 & 182 & 167 & 163 \\
\hline
\end{tabular}

Experiments with varying partial pressures of hydrogen chloride and carbon dioxide indicate that the increase in intensity is proportional to the partial pressure of the foreign gas.

The ultra-violet absorption continuum of bromine between $3300 \mathrm{~A}$. and $2500 \mathrm{~A}^{2}$ is affected to a much greater extent, the intensity being increased as much as ten-fold by $60 \mathrm{~cm}$. of hydrogen chloride or bromide. In this region, numerical values of the absorption coefficient may have little meaning, since in the presence of oxygen they are not independent of the partial pressure of bromine.

N. S. BAyLiss.

University of Western Australia.

A. L. G. REes.

University of Melbourne. Jan. 27.

${ }^{1}$ Acton, Aickin and Bayliss, J. Chem. Phys., 4, 474 (1936). ${ }^{2}$ Aickin and Bayliss, Trans. Far. Soc., 84, 1371 (1938).

\section{Theory of Mesons and Nuclear Forces}

IN a recent note ${ }^{1}$ on the static interaction between nuclear particles, Dr. C. Møller and Prof. L. Rosenfeld describe the results of a canonical transformation which makes it possible to separate dynamic and static interactions, and they state: "The static interaction is in all cases exactly the same as that obtained as a first approximation in the perturbation method". They referred to certain work done previously ${ }^{2}$ by 